\title{
Reporting uncertainty in results-Response to article on copper cold-spray technology
}

\author{
Piyali Chatterjee $\mathrm{PhD}^{1}$, John D. Coppin $\mathrm{MPH}^{1}$, Marjory D. Williams PhD, RN ${ }^{1}$, Hosoon Choi PhD ${ }^{1}$, John E. Zeber PhD ${ }^{1,2}$, \\ Richard E. Nelson $\mathrm{PhD}^{3,4}$ and Chetan Jinadatha MD, MPH ${ }^{5}$ (1) \\ ${ }^{1}$ Department of Research, Central Texas Veterans Healthcare System, Temple, Texas, ${ }^{2}$ Department of Health Promotion and Policy, School of Public Health and \\ Health Sciences, University of Massachusetts, Amherst, Massachusetts, ${ }^{3}$ IDEAS Center, Veterans' Affairs Salt Lake City Healthcare System, Salt Lake City, Utah, \\ ${ }^{4}$ Division of Epidemiology, University of Utah School of Medicine, Salt Lake City, Utah and ${ }^{5}$ Department of Medicine, Central Texas Veterans Health Care System, \\ Temple, Texas
}

To the Editor-Antimicrobial surfaces are an important tool in fighting healthcare-associated infections. The article by Lucas et al. ${ }^{1}$ in a recent issue of Infection Control and Healthcare Epidemiology (ICHE) describes the evaluation of the effectiveness of a cold-spray method of copper-coated surface on reducing bioburden. A comparison of different methods of copper coatings (ie, the plasma spray, copper-wire arc method, and the cold-spray method) suggests that the cold-spray method was the most effective due to the high dislocation density and diffusivity of the coldspray copper coatings, ${ }^{2}$ which are necessary for bacteriostatic action of copper. Although the results generated by Lucas et al suggest that the cold-spray method may hold promise, the study has several methodological limitations that might detract from their findings and weaken the argument that this surface type is more effective. We discuss them here.

The Environmental Protection Agency (EPA) protocol for testing of copper surface products states that multiple carriers and a soil load should be included in these studies and that reductions should be calculated by comparing controls to treated carriers. Based on these guidelines set forth by EPA, the data presented by Lucas et al. are inadequate to claims the effectiveness of this product with certainty because different pathogens were used on only 1 size of carrier. Also, these researchers did not report using organic soil load in the sample preparations, which are generally used for mimicking real-world hospital surfaces. More importantly, although $>7 \log$ microbial killing was reported, it was not clear whether desiccation was taken into consideration in these experiments, which complicates interpretation of the actual log-kill data presented. The biocidal efficacy of the copper coating tested on a dry inoculum commonly found on hospital surfaces is more useful instead of the wet liquid suspension method described. ${ }^{3}$ In addition to antimicrobial efficacy, the EPA protocol also tests durability (abrasion and chemical exposure) of the test material as a major component of the testing. The durability of this material was not reported, and these data would have provided valuable information on the efficacy of this product for long-term use as would comparisons with other copper or copper-alloy embedded products in a real-world setting.

Finally, while reporting data, the EPA protocol also recommends that geometric means are calculated for 3 control and 5 test carriers and then a percentage of reduction is calculated. Although it is difficult to decipher from the methods reported, it appears that only a single sample across time points per organism and test material type was used. Furthermore, uncertainty in the form of standard errors of their regression model or standard deviations is not reported in their data. Thus, the reader must assume either that an omission was made concerning the statistical uncertainty in their data and model estimates or that only a single sample was used and thus no variation could be reported. Also, no shaded confidence or error regions are provided in the figures. In addition, $\mathrm{R}^{2}$ is described but the values are not reported. Without these supporting data, it is impossible to determine the overall results of the experiment. Other researchers would be very interested in seeing the full results of their regression models, including all parameter estimates, standard errors, and $\mathrm{R}^{2}$ data.

Although the findings described in the study by Lucas et al. are promising because the cold-spray technique as described can reduce bioburden in a very short time period (15 minutes) compared to solid copper surfaces (3 hours), we are unable to determine whether these findings are meaningful based on the methods and results described. We hope that the authors will make their data available and will provide more detailed description of the methods and the results from their analyses. Future studies on cost-effectiveness and durability of the product are also needed for evaluation for practical use and widespread commercialization.

Acknowledgments. The views expressed in this article are those of the author(s) and do not necessarily represent the views of the Department of Veterans' Affairs.

Financial support. This study was supported by funding from the Agency for Healthcare Research and Quality (AHRQ grant no. 1R01HS025598-01A1).

Conflicts of interest. All authors report no conflicts of interest relevant to this article

\section{References}

1. Lucas MDI, Botef I, Reid RG, van Vuuren SF. Laboratory-based study of novel antimicrobial cold-spray coatings to combat surface microbial contamination. Infect Control Hosp Epidemiol 41:1378-1383.

2. Champagne, VK, Helfritch, DJ. A Demonstration of the antimicrobial effectiveness of various copper surfaces. J Biol Eng 2013;7:8.

3. McDonald M, Wesgate R, Rubiano M, Holah J, Denyer SP, Jermann C, Maillard J-Y. Impact of a dry inoculum deposition on the efficacy of copper-based antimicrobial surfaces. J Hosp Infect 2020;106:465-472.

\footnotetext{
Author for correspondence: Chetan Jinadatha, E-mail address: chetan.jinadatha@ va.gov

Cite this article: Chatterjee $\mathrm{P}$, et al. (2022). Reporting uncertainty in results—Response to article on copper cold-spray technology. Infection Control \& Hospital Epidemiology, 43: 537, https://doi.org/10.1017/ice.2021.32
}

(c) The Author(s), 2021. Published by Cambridge University Press on behalf of The Society for Healthcare Epidemiology of America. 